\title{
Infección Postoperatoria en cirugía ginecológica mayor por vía vaginal
}

\section{Dr. Alvaro Caicedo $\mathrm{H}$ *}

Son muchos los factores que tienen influencia en la infección ocurrida en el postoperatorio. Principalmente hay que considerar el agente causal, la distribución y origen de éste además de la suceptibilidad del huesped.

Lo fundamental para toda infección es su prevención y de no ser posible lo anterior, el tratamiento específico para cada tipo de germen. Aunque se ha progresado mucho en lo concerniente al laboratorio para determinar gérmenes principalmente anerobios y virus, en el campo de antibióticos, antisépticos y medios físicos; por su parte los gérmenes también han progresado en crear resistencia a las armas de combate que la medicina usa contra ellos.

Los gérmenes encontrados en la vagina son muy diversos y van desde virus hasta protozoarios incluyendo bacterias anaeróbicas y aeróbicas, especies de microplasmas y hongos. Los lactobacilos (Bacilos de Doderlein) son importantes para mantener el $\mathrm{PH}$ vaginal y prevenir la sobreproducción de algu- nos gérmenes, principalmente enterobacteriaceas (1). Algunos antibióticos alteran la flora normal logrando combatir algunos gérmenes, pero a su vez permitiendo la proliferación de otros por alteración del $\mathrm{PH}$.

A continuación se puede leer la lista de gérmenes que hacen parte de la flora vaginal. Aunque algunos organismos son potencialmente patógenos pueden encontrarse sin que haya evidencia de infección (1).

- Estafilococo epidermis

- Estafilococo aureas

- Especies de peptococos

- Bifidobacterium

- Enterobacterium

- Enterobacteriaceas.

- Estreptococo Viridans

- Estreptococos A. B. C. D. G.

- Estreptococos Microaerofílico

- Peptoestreptococos

- Neiseria

- Veillonedas

- Lactobacilos

- Hemofilus Vaginalis

\begin{tabular}{|c|c|c|c|}
\hline \multicolumn{4}{|c|}{ CUADRO No. 1} \\
\hline & GERMENES & CERVIX & VAGINA \\
\hline & Aerobios & $37^{\circ} \%$ & $34^{\mathrm{o}} / \mathrm{o}$ \\
\hline & Lactobacilos & $10 \%$ & $9 \%$ \\
\hline & Corinebacteria & $6 \%$ & $6 \%$ \\
\hline & Estreptococo & $13 \%$ & $11 \%$ \\
\hline & Estafilococo epidermis & $3 \%$ & $1 \%$ \\
\hline & Enterobacteriaceas & $2 \%$ & $3 \%$ \\
\hline & Anaerobios & $31 \%$ & $35 \%$ \\
\hline - & Peptococci & $10 \%$ & $9 \%$ \\
\hline - & Peptoestreptococcia & $2 \%$ & $2 \%$ \\
\hline- & Bacilos gram positivos & $12 \%$ & $13 \%$ \\
\hline & Lactobacilos & $4 \%$ & $4 \%$ \\
\hline & Eubacterias & $2 \%$ & $5 \%$ \\
\hline & Clostridium & $2 \%$ & $2 \%$ \\
\hline- & Bacteroides & $7 \%$ & $7 \%$ \\
\hline & Cándida & $2 \%$ & $2 \%$ \\
\hline & (Al & $.15 / 78 \mathrm{P}$ & \\
\hline
\end{tabular}

* Del Depto. de Obstetricia y Ginecologia Hospital de San José, Bogotá, D.E.
(The Medical Clinics of North America, Junio 1974) 
Estudios realizados por John G. Bartlett y Col en cultivos aislados de endocervix y fondo de saco posterior tanto para aerobios como para anaerobios, demostraron que los gérmenes de éstos dos sitios son similares, encontrándose algunas diferencias tan solo en casos aislados. Los resultados pueden verse a continuación: (2) CUADRO No. 1

Casi todos los gémenes encontrados en la piel y en el tacto genital pueden ser causantes de infección.

La siguiente es la lista de organismos causantes de infección en ginecología y.obstetricia.

\section{MICROORGANISMOS CAUSANTES DE INFECCION EN GINECOLOGIA Y OBSTETRICIA (1)}

\section{VULVOVAGINITIS}

- Infecciones venéreas:

N. Gonorreae - treponema palidum - Clamydia del linfogranuloma venéreo. Hemofilus Ducrey y Donovania.

- S. Aureus

- S. Epidermis

- Estreptococos

- Cándidas

- Herpes virus hominis

- $\mathrm{H}$. vaginalis

- Tricomonas vaginalis

\section{CERVICITIS}

- N. gonorreae

- S. Aureus

- Estreptococos

- Enterobacteriaceas

- Mycrobacterium Tuberculosis

- Herpes virus hominis

- Cytomegalavirus

- T. vaginalis

- C. albicens

\section{ENDOMETRITIS}

- N. Gonorreaè

- Estreptococos

- Estafilococos
- Enterobacteriaceas

- Especie de bacteroides

- Clostridium perfringens

- M. Tuberculosis

\section{SALPINGITIS - OFORITIS}

- Estreptococos

- S. Aureus

- Enterobacteriaceas

- Especies de bacteroides

- N. Gonorreae

- M. Tuberculosis

\section{BACTEREMIA}

- N. gonorreae

- Estreptococos

- S. aureus

- Enterobacteriáceas

- Bacilos anaerobicos gram negativos (tales como Bacterioides fragilis).

- Clostridium Welchii

- H. vaginalis

- Especies de Mycoplasmas

(The Medical Clinies of North America, Julio 1974)

\section{OBJETIVO}

En vista de la alta incidencia de infección postoperatoria en cirugía vaginal ginecológica, en el Departamento de Ginecología y Obstetricia del Hospital San José de Bogotá, la falta de respuesta de las infecciones al uso de la Ampicilina (Antibiótico de uso muy amplio en el servicio de ginecología) y el elevado número de días cama por paciente que ésta morbilidad representa; se resuelve investigar sesenta pacientes del servicio asistencial admitidas por patología ginecológica mayor, para ser intervenidas por vía vaginal.

\section{MATE RIAL Y METODOS}

Se escogen tres grupos cada uno de veinte pacientes los cuales se denominan con las letras A. B. y C. admitidos para cirugía ginecologíca mayor por vía vaginal desde Enero de 1977 a Marzo de 1978.

A las pacientes del Grupo A, se les adminis- 
tró Ampicilina por vía oral, $500 \mathrm{mcg}$. cada seis horas durante un mínimo de tres días antes de la cirugía y no se administraron antibióticos de la intervención a no ser por causa mayor. El grupo B, recibió Ampicilina $500 \mathrm{mg}$. por vía oral o intravenosa cada 6 horas a partir del post-operatorio inmediato durante un mínimo de cinco días, y al grupo $\mathrm{C}$ no se le dió ningún antibiótico durante el pre o postoperatorio.

Todas las pacientes fueron sometidas a exámenes preoperatorios de rutina para el hospital y se aceptaron para el estudio aquellas mujeres libres de infección de cualquier índole, y con urocultivos negativos.

Las pacientes que por una u otra razón presentaban algún riesgo por el no uso de antibióticos eran formuladas con el tratamiento adecuado y no se incluyeron en el estudio.

\section{RESULTADOS}

Edad:

La edad promedio del grupo en estudio fue 55.15 siendo la menor 30 años y la mayor 71. La edad promedio para los grupos A. B. y C. fue muy similar, como se puede 'ver en la tabla No. 1.
No. 1 TABLA DE EDADES (años)

GRUPOS

EDAD PROMEDIO

\begin{tabular}{|c|c|}
\hline $\begin{array}{l}\mathrm{A} \\
\mathrm{B} \\
\mathrm{C}\end{array}$ & $\begin{array}{l}55.95 \\
53.35 \\
53.15\end{array}$ \\
\hline TOTAL PROMEDIO & 53.15 \\
\hline
\end{tabular}

\section{Diagnóstico:}

Los diagnósticos de ingresos fueron los mismos de postoperatorio. (No se tuvo en cuenta diagnóstico Histopatológicos).

Los tres diagnósticos más frecuentes fueron: Cisto-Recto-Histerocele (prolapso conjugado) grado III, Cisto-Reetocelo grado II, y la combinación de Cistocele grado II con Rectocele grado III (Ver cuadro No. 2).

\section{Intervenciones quirúrgicas:}

El $50 \%$ de las cirugías practicadas fueron Colpoperineorrafias, seguidas de Histerectomias Vaginales $(43.33 \%)$, operación de Kelly $(5 \%)$, y una Colpocleisis para corrección de un prolapso de cúpula. Ver cuadro No. 3.

\begin{tabular}{|c|c|c|c|c|c|}
\hline \multicolumn{6}{|c|}{$\begin{array}{l}\text { CUADRO No. } 2 \\
\text { DIAGNOSTICOS }\end{array}$} \\
\hline Diagnósticos & A & B & C & No. & $\%$ \\
\hline $\begin{array}{l}\text { Cistocele } \\
\text { I }\end{array}$ & & 1 & & 1 & 1.66 \\
\hline $\begin{array}{l}\text { Cistocele II } \\
\text { Rectocele II }\end{array}$ & & 1 & 1 & 2 & 3.33 \\
\hline $\begin{array}{l}\text { Cistocele I } \\
\text { Rectocele III }\end{array}$ & & & 1 & 1 & 1.65 \\
\hline $\begin{array}{l}\text { Cistocele II } \\
\text { Rectocele II }\end{array}$ & 9 & 4 & 10 & 23 & 38.33 \\
\hline $\begin{array}{l}\text { Cistocele II } \\
\text { Rectocele III }\end{array}$ & 2 & 2 & & 4 & 6.66 \\
\hline $\begin{array}{l}\text { Prolapso } \\
\text { Conj. III }\end{array}$ & 9 & 12 & 7 & 28 & 46.66 \\
\hline $\begin{array}{l}\text { Prolapso } \\
\text { Cúpula }\end{array}$ & & 1 & & 1 & 1.66 \\
\hline TOTAL & 20 & 20 & 20 & 60 & 99.99 \\
\hline
\end{tabular}




\begin{tabular}{|l|r|r|r|r|r|}
\hline \multicolumn{7}{|c|}{ CUADRO No. 3 } \\
\hline CIRUGIA PR ACTICADA \\
\hline Operación & A & B & C & No. & \% \\
\hline Kelly & 1 & 1 & 1 & 3 & 5 \\
Histerectomia & 9 & 9 & 12 & 30 & 50 \\
Vaginal & 10 & 10 & 6 & 25 & 1.33 \\
Colpocleisis & & & 1 & 1 & 99.99 \\
\hline TOTAL & 20 & 20 & 20 & 60 & \\
\hline
\end{tabular}

\section{Estancia:}

Los días camas ocupados por las pacientes en el tiempo pre-operatorio fue de 7.5 días, lo cual es muy elevado si se compara con otras instituciones o incluso pacientes particulares con diagnósticos iguales intervenidas en esta misma institución. Para cada uno de los tres grupos, el número de días pre-operatorio fue similar. Ver cuadro No. 4.
Los días, durante el post-operatorio fueron de 10.65 días, lo cual también es elevado, siendo mayor para el grupo A (Antibiótico Preoperatorio), seguido del C (Antibiótico Postoperatorio) y del B (No antibiótico). Ver cuadro No. 4.

\section{Urocultivos pre y post-operatorios:}

Teniendo en cuenta los urocultivos, todos

\section{CUADRO No. 4}

DIAS CAMA DURANTE EL PRE Y POST-OPERATORIO

\begin{tabular}{|l|c|c|c|c|}
\hline & & & & \multicolumn{1}{|c|}{ TOTAL } \\
\hline Promedio Días/cama & $\mathrm{A}$ & $\mathrm{B}$ & 7.3 & 7.58 \\
Preoperatorio & 8.5 & 6.95 & 10.0 & 10.66 \\
\hline
\end{tabular}

CUADRO No. 5

GERMENES Y SUS ASOCIACIONES EN UROCULTIVOS

(POST-OPERATORIO)

\begin{tabular}{|l|r|r|r|r|}
\hline GERMENES & \multicolumn{1}{|c|}{$\mathrm{A}^{\mathrm{o}}$} & \multicolumn{1}{c|}{$\mathrm{B}^{\mathrm{o}} \mathrm{o}$} & \multicolumn{1}{c|}{$\mathrm{C}^{\mathrm{o}}$} & \multicolumn{1}{c|}{ TOTAL $\%$} \\
\hline Citrobacter & 35,00 & 31.57 & 38.88 & 35.08 \\
E. Coli & 45.00 & 21.05 & 27.77 & 31.57 \\
Enterobacter & 5.00 & 26.31 & 5.55 & 12.28 \\
Proteus & 5.00 & 5.26 & 16.66 & 8.77 \\
Proteus/E. Coli & 5.00 & & 5.55 & 3.50 \\
Enterobacter/E. Coli & 5.00 & 5.26 & & 1.75 \\
Para Coli/E. Coli & & 5.26 & & 1.75 \\
Proteus/Enterobacter & & & 5.55 & 1.75 \\
Enterobacter/E. Coli & & 5.26 & & 1.75 \\
Seudomona/Citrobacter & & & & 1.75 \\
Klebsiella & & & 99.96 & 99.95 \\
\hline TOTAL & 100.00 & 99.99 & & \\
\hline
\end{tabular}


fueron negativos en el preoperatorio, condición para aceptar a la paciente en el estudio. Por otra para los urocultivos tomados en el post-operatorio después de retirada la sonda de Foley, fueron positivos en el 95 \% de los casos con recuento de colonias superior a 200.000. Teniendo en cuenta cada uno de los grupos, para el A ( bióticos en el post-operatorio) fue del 100 $\%$, para el C (no recibió antibióticos) fue del $90 \%$, y para el grupo B fue intermedio entre los anteriores $95 \%$.

Los gérmenes encontrados en los cultivos de orina fueron principalmente: E. Coli $(35.38 \%)$. Clases de Citrobacter $(32.30$ $\%$ o). Enterobacter (15.38) y Proteus (12.30 o). Klebsiela, Pseudomona y para Coli fueron hallados en casos aislados. Algunos cultivos mostraron asociaciones de dos ó más gérmenes, sin importancia estadística. Ver cuadro No. 5 .
Analizando por grupos el A y C presentaron un $35 \%$ de infección cada uno, y en el grupo B solo ocurrió en un $10 \%$ lo cual puede ser indicativo de la acción del antibiótico en el post-operatorio. Ver cuadro No. 7 .

\begin{tabular}{|c|c|c|}
\hline \multicolumn{3}{|c|}{ CUADRO No. 7} \\
INFECCION VAGINAL \\
\hline GRUPOS & No. DE CASOS & $\%$ \\
\hline A & 7 de 20 & 35 \\
B & 2 de 20 & 10 \\
C & 7 de 20 & 35 \\
\hline TOTAL & 16 de 60 & 26 \\
\hline
\end{tabular}

Gérmenes en cultivos vaginales en el postoperatorio:

\begin{tabular}{|l|c|c|c|c|}
\hline \multicolumn{5}{|c|}{ CUADRO No. 6} \\
\hline PORCENTAJE DE CASOS PARA CADA GERMEN \\
\hline GERMENES & $\mathrm{A} \%$ & $\mathrm{~B} \%$ & $\mathrm{C}^{\mathbf{0}} \%$ & TOTAL $\%$ \\
\hline Citrobacter & 31.81 & 28.57 & 36.36 & 32.30 \\
E. Coli & 50.00 & 23.80 & 31.81 & 35.38 \\
Proteus & 9.09 & 9.52 & 18.18 & 12.30 \\
Enterobacter & 9.09 & 28.57 & 9.09 & 15.36 \\
Klebsiella & & 4.76 & 4.54 & 1.53 \\
Seudomona & & 4.76 & & 1.53 \\
Paracoli & 99.99 & 99.99 & 99.99 & 99.99 \\
\hline TOTAL & & & & \\
\hline
\end{tabular}

El cuadro No. 6, muestra el porcentaje de casos para cada gérmen, sin tener en cuenta las asociaciones.

Analizando los grupos, en particular el grupo A, presentó más infecciones por E. Coli que por Citrobacter siendo éste último, el de más incidencia para el grupo B y C, En el grupo $C$, se encontraron más casos de Proteus, y en el grupo B, mayor incidencia de Enterobacter con relación a A y C.

\section{Infección vaginal post-operatoria:}

Teniendo en cuenta el total de los casos, la infección vaginal se presentó en el $26 \%$.
El germen encontrado en los tres grupos fue E. Coli $(33.33 \%)$ principalmente en el grupo A $(42.85 \%)$.

Proteus apareció tanto en el grupo A $(42.85 \%$ o $)$ como en el C $(12.50 \%$ o $)$ para un total de $22.22 \%$ o.

Enterococo y Enterobacter solo ocurrieron en el Grupo B.

Estafilococo y Pseudomona îue visto en el grupo C solamente.

El Grupo B fue el menos afectado, lo cual puede ser efecto del uso de antibiótico en el post-operatorio. En los grupos $\mathrm{A} \mathrm{y} \mathrm{C} \mathrm{hu-}$ bo dos casos de infección vaginal evidente 
por clínica con cultivo negativo. Ver cuadro No. 8.
Antibióticos tales como Penicilina, Amoxacilina, Cefalosporina, Tetraciclina y Acido

\begin{tabular}{|c|c|c|c|c|}
\hline \multicolumn{5}{|c|}{ CUADRO No. 8} \\
\hline GERMEN & $\mathrm{A} \%$ & $\mathrm{~B} \%$ & $\mathrm{C}^{\mathrm{O}} \%$ & TOTAL $\%$ \\
\hline $\begin{array}{l}\text { Proteus } \\
\text { E. Coli } \\
\text { Enterococo } \\
\text { Enterobacter } \\
\text { Estafilococo } \\
\text { Hemolítico } \\
\text { Pseudomona } \\
\text { Negativos }\end{array}$ & $\begin{array}{r}42.85 \\
42.85 \\
\\
\\
14.28\end{array}$ & $\begin{array}{l}33.33 \\
33.33 \\
33.33\end{array}$ & $\begin{array}{l} \\
37.50 \\
12.5 \\
12.50\end{array}$ & $\begin{array}{r}22.22 \\
33.33 \\
5.55 \\
5.55 \\
\\
16.66 \\
5.55 \\
11.11\end{array}$ \\
\hline
\end{tabular}

\section{Antibiogramas:}

Los estudios de los antibiogramas tanto para la infección urinaria como vaginal, no fueron uniformes por falta de realizar las pruebas de sensibilidad con los mismos antibióticos en todos los casos, por éste motivo solo seleccionamos aquellos antibióticos más utilizados en pruebas de sensibilidad en el laboratorio y los de fácil obtención en el hospital. Los resultados se muestran en el cuadro No. 9
Oxolinico, fueron poco utilizados en las pruebas de antibiogramas por lo cual no se tomaron en consideración.

Los resultados de los antibiogramas tomados para las secreciones vaginales fueron las siguientes (ver cuadro No. 10).

Los comentarios referentes a los antibiogramas (Cuadro No. 9 y 10) se hacen más objetivos y obvios en las gráficas correspondientes.

\section{CUADRO No. 9}

RESULTADOS DE ANTIBIOGRAMAS EN ORINA

\begin{tabular}{|c|c|c|c|c|}
\hline MEDICAMENTO & $\mathrm{A} \%$ & $\mathrm{~B} \%$ & $\mathrm{C} \%$ & TOTAL $\%$ \\
\hline Ampicilina & $\begin{array}{l}\text { R-90.00 } \\
\text { S-10.00 }\end{array}$ & $\begin{array}{l}\mathrm{R}-100 \\
\text { S- }-\end{array}$ & $\begin{array}{l}\mathrm{R}-82.35 \\
\mathrm{~S}-17.65\end{array}$ & $\begin{array}{l}R-91.00 \\
\text { S- } 9.00\end{array}$ \\
\hline Cloranfenicol & $\begin{array}{l}R-73.33 \\
\text { S-26.60 }\end{array}$ & $\begin{array}{l}\text { R-66.66 } \\
\text { S.33.33 }\end{array}$ & $\begin{array}{l}R-60.00 \\
\text { S- } 40.00\end{array}$ & $\begin{array}{l}R-66.66 \\
\text { S-33.33 }\end{array}$ \\
\hline Garamicina & $\begin{array}{l}\text { R-22.22 } \\
\text { S-77.77 }\end{array}$ & $\begin{array}{l}R-23.52 \\
\text { S-76-47 }\end{array}$ & $\begin{array}{l}\mathrm{R}-\overline{-} \\
\mathrm{S}-100.00\end{array}$ & $\begin{array}{l}R-15.69 \\
\text { S-84-31 }\end{array}$ \\
\hline Kanamicina & $\begin{array}{l}R-68.75 \\
\text { S-31-25 }\end{array}$ & $\begin{array}{l}R 47.05 \\
\text { S-52.94 }\end{array}$ & $\begin{array}{l}R-18.75 \\
\text { S-81-25 }\end{array}$ & $\begin{array}{l}\text { R-49.89 } \\
\text { S-50-10 }\end{array}$ \\
\hline Mandelamine & $\begin{array}{l}R-5.00 \\
\text { S-95.00 }\end{array}$ & $\begin{array}{l}R-10.00 \\
\text { S-90.00 }\end{array}$ & $\begin{array}{l}\mathrm{R}-- \\
\mathrm{S}-100.00\end{array}$ & $\begin{array}{l}R-5.36 \\
S-94.64\end{array}$ \\
\hline Bactrim & $\begin{array}{l}\mathrm{R}-27.77 \\
\mathrm{~S}-72.22\end{array}$ & $\begin{array}{l}R-15-39 \\
\text { S-84.61 }\end{array}$ & $\begin{array}{l}R-41.67 \\
\text { S-58.33 }\end{array}$ & $\begin{array}{l}\text { R-27.91 } \\
\text { S-72.09 }\end{array}$ \\
\hline
\end{tabular}


CUADRO No. 10

RESULTADOS DE ANTIBIOGRAMAS EN INFECCION VAGINAL

\begin{tabular}{|c|c|c|c|c|}
\hline MEDICAMENTO & $\mathrm{A} \%$ & $\mathrm{~B} \%$ & $\mathrm{C}^{\mathrm{O}} \%$ & TOTAL $\%$ \\
\hline Ampicilina & $\begin{array}{l}\text { R-50.00 } \\
\text { S-50.00 }\end{array}$ & $\begin{array}{l}\text { R-50.00 } \\
\text { S-50.00 }\end{array}$ & $\begin{array}{l}\mathrm{R}-20.00 \\
\mathrm{~S}-80.00\end{array}$ & $\begin{array}{l}\text { R-38.47 } \\
\text { S-61.53 }\end{array}$ \\
\hline Cloranfenicol & $\begin{array}{l}R-- \\
\text { S-100.00 }\end{array}$ & $\begin{array}{l}R-50.00 \\
\text { S-50.00 }\end{array}$ & $\begin{array}{l}\mathrm{R}-\overline{-} \\
\mathrm{S}-100.00\end{array}$ & $\begin{array}{l}\text { R-10.00 } \\
\text { S-90.00 }\end{array}$ \\
\hline Penicilina & $\begin{array}{l}\mathrm{R}-- \\
\mathrm{S}-100.00\end{array}$ & $\begin{array}{l}\mathrm{R}-- \\
\mathrm{S}-100.00\end{array}$ & $\begin{array}{l}\mathrm{R}-100.00 \\
\mathrm{~S}-\mathrm{C}\end{array}$ & $\begin{array}{l}R-20.00 \\
\text { S-80.00 }\end{array}$ \\
\hline Garamicina & $\begin{array}{l}\text { R- - } \\
\text { S-100.00 }\end{array}$ & $\begin{array}{l}R-50.00 \\
\text { S-50.00 }\end{array}$ & $\begin{array}{l}\mathrm{R}-\overline{ } \\
\mathrm{S}-100.00\end{array}$ & $\begin{array}{l}\text { R-10.00 } \\
\text { S-90.00 }\end{array}$ \\
\hline Bactrim & $\begin{array}{l}\mathrm{R}-17.77 \\
\mathrm{~S}-83.33\end{array}$ & $\begin{array}{l}\text { No hay } \\
\quad \text { dato } \\
\text { No hay } \\
\text { dato }\end{array}$ & $\begin{array}{l}\text { R- } \\
\text { S-100.00 } \\
\text { S-100.00 }\end{array}$ & $\begin{array}{l}\text { R-10.00 } \\
\text { S-90.00 } \\
\text { S-90.00 }\end{array}$ \\
\hline Tetraciclina & $\begin{array}{l}\text { S-100.00 } \\
\text { R- - }\end{array}$ & $\begin{array}{l}\text { No hay } \\
\text { dato } \\
\text { No hay } \\
\text { dato }\end{array}$ & $\begin{array}{l}\text { S-100.00 } \\
\text { R- - }\end{array}$ & $\begin{array}{l}\text { S-100.00 } \\
\text { R- - }\end{array}$ \\
\hline Kanamicina & $\begin{array}{l}\text { R- - } \\
\text { S-100.00 }\end{array}$ & $\begin{array}{l}\mathrm{R}-- \\
\text { No hay } \\
\text { dato }\end{array}$ & $\begin{array}{l}\mathrm{R}-- \\
\mathrm{S}-100.00\end{array}$ & $\begin{array}{l}R-- \\
\text { S-100.00 }\end{array}$ \\
\hline
\end{tabular}

TABLA DE EDADES ( $\mathrm{AÑOS})$

\begin{tabular}{|l|c|}
\hline GRUPOS & PROMEDIO \\
\hline A & 55.95 \\
B & 53.35 \\
C & 52.15 \\
\hline Promedio Total & 53.15 \\
\hline
\end{tabular}

\section{Sonda de Foley:}

En toda paciente para éste estudio se aplico sonda de Foley. El tiempo de uso de la sonda de Foley, puede ser calculado en más o menos de 6 a 7 días, encontrándose casos de más de 20 días. El cálculo exacto no puede ser tomado debido a la falta de datos en las historias.

\section{CONCLUSIONES}

Para los tres grupos, las edades fueron simi- lares lo cual nos muestra un grupo muy homọgeneo. Teniendo en cuenta los diagnósticos no podemos concluir igualdad para los grupos, pero al considerar las intervenciones se encuentra estrecha similitud entre ellos .Estas edades son similares a las encontradas por el Dr. Arturo Rodriguez Soto (3).

Para el total de los grupos, los días cama durante el pre-operatorio,fueron muchos, ya que lo corriente en otros hospitales y aún en pacientes particulares del mismo hospital, con los mismos diagnósticos y sometidos a intervenciones iguales a las del grupo estudiado; no completan las 24 horas de hospitalizadas antes de la intervención. El número pues de días cama pre-operatorio promedio obtenido en éste estudio (7.5 días), equivale casi a los días de un paciente en post-operatorio. Esta irregularidad en la organización hospitalaria como es lógico, trae disminución del número de cirugías al año y un costo más elevado para el hospital, además de no pocos inconvenientes socio-económicos para las pacientes. 
El número de días cama post-operatorio por paciente está influído por una alta morbilidad y se observa que es mayor tanto en el grupo que recibió antibiótico profilactico en el pre-operatorio únicamente, como en aquel que no se le administró nịngún antibiótico. Tal promedio de 10.6 días para los tres grupos, es muy elevado, comprometiendo factores socio-económicos tanto del paciente como del hospital.

La positividad de los urocultivos en el postoperatorio con recuentos de colonias de más de 200.000 fueron muy altos para todos los grupos, solamente un $5 \%$ de diferencia para el grupo C y un $10 \%$ para el grupo B con relación al grupo A que fue el $100 \%$ o. De éstos resultados se confirma lo mencionado en casi todos los textos de medicina relacionados al uso de sondas vesicales las cuales inevitablemente predisponen a la infección de vías urinarias. (5) (6).

El tiempo de permanencia de la sonda fue imposible de determinar para todos los casos tal como es mencionado ya por otros autores (6).

En éste estudio, se comprueba que la infección no se previene con el uso de ampicilina y en cambio puede suceder que el uso de. dicho antibiótico llegue a producir cambios de la flora normal, que favorecerian la infección qe se busca prevenir.

Relacionando los tres grupos, de acuerdo a los gérmenes que causaron la infección urinaria, se observa que el grupo $\mathrm{A}$ fue el de mayor incidencia de los tres grupos y donde más se presentó Citrobacter y E. Coli.

Para el Grupo B el porcentaje de gérmenes fue menor que en A y C, excepto para Enterobacter.

El grupo C mostró mayor incidencia para Citrobacter con relación a los otros grupos.

La infección vaginal post-operatoria ocurrió en más de la cuarta parte del grupo estudiado, pero con cifras significativas para los dos grupos sin antibióticos durante el post-operatorio, lo cual demuestra que el uso del antibiótico después de cirugía puede pasar a ser una forma terapéutica y no profilática.

Los gérmenes de mayor incidencia en la in- fección vaginal fueron É. Coli y Proteus, o sea los mismos gérmenes causantes de la mayoría de infecciones encontradas en la orina. Esto demuestra contaminación a partir de un solo origen. Para los doctores A. Jubis y S. Velez fue más frecuente la Klebsiella y en segundo lugar el Coli.

El análisis de los antibióticos demuestra que la ampicilina no es el antibiótico de elección, ni profilactico, ni terapéutico para la infección urinaria, ocurrida después de cirugía vaginal, al igual que el cloranfenicol con un $66.66 \%$ o de resistencia. La Kanamicina mostró resistencia en el $50 \%$. El antibiótico que demostró ser más sensible fue la Mandelamine $(94.64 \%$ ) (6) seguido de la Garamicina ( $84.31 \%$ ) y Bactrím ( 72 . $09 \%$ ).

Para infección vaginal la ampicilina mostró un poco más del $50 \%$ de sensibilidad, siendo inferior a otros antibióticos, como el cloranfenicol, garamicina y penicilina.

\section{RECOMENDACIONES}

- El uso de sonda de Foley debe ser lo más corto posible y siempre conectada a cistoflow o a bocal estéril y sellado (4).

- De acuerdo a éste estudio al antibiótico utilizado en el post-operatorio de cirugía ginecológica por vía vaginal, no es profiláctica sino terapeutico por lo cual se debe usar a dosis adecuadas.

- La ampicilina no es el antibiótico ideal para éste tipo de cirug1a y si se emplea se debe utilizar en combinación con otro antibiótico.

- Las drogas de elección según se demuestra en éste trabajo pueden ser: garamicina, mandelamine, bactrim, Kanamicina.

\section{RESUMEN}

Se estudiaron tres grupos de pacientes hospitalizados para cirugía ginecológica mayor denominados con las letras A, B y C.

Al grupo A se le administró ampicilina preoperatoria.

Al B Ampicilina post-operatoria y al $\mathrm{C}$ no 
se le da antibióticos.

Se concluye que en todos los grupos hubo infección urinaria después de retirar la sonda de Foley con alta resistencia al antibiótico usado.

La infección vaginal post-operatoria ocurrió en más de la cuarta parte de las pacientes.

Se concluyó que el antibiótico utilizado no es adecuado para éste tipo de intervención y que todo antibiótico utilizado en cirugía ginecológica, debe ser con fines terapéuticos y no profilácticos.

\section{SUMMARY}

The purpose of this study is to analyze the frecuency of post-operation infection in major gineacological surgery through the vagina.

The cases studied in the Gineacology and Obstetrics Departament of Bogotá's Hospital de San José were classified in three groups: The first group, or Group A, was treated with ampiciline before the operation; the second one, or Group B, with ampiciline after the operation, and the third group, or
Group C, was not treated at any time with antibiotics. It was noticed that all the groups suffered from urinary infection with high resistance to the antibiotic used after removing Foley's Probe, and that more than $25 \%$ of the pacients had post-operation vaginal infection. Hence, it is concluded that the antibiotic used is not suitable for this type of operations and that all antibiotic used in gineacological surgery should have therapeutic and not prophylactic purposes.

\section{BIBLIOGRAFIA}

(1) The Medical Clinics of North America Julio 1974

(2) Bartlett John G. AMA. J. OB/GYN. Mar 15/ $78-650$.

(3) Rodriguez Soto Arturo Histerectomías. Estudio Comparativo de 1000 operaciones. Rev Col OB/GYN Jul - Ago 1961 - 40 - 49

(4) Watts y Kimbrough - Hysterectomy Analysis of 1000 consectuvie Operation OB/GYN 7 : 4831956.

(5) Gillespie WA. Lennon Prevention of Catheter Infection of Urine in Female Patient. British Med 5 - 11 - 121962 .

(6) Jubis Alfonso. Velez Santiago. Estudio sobre Infección urinaria en Ginecología Rev. Col. OB/GIN. Vol. 20 No. 21969. 